\title{
Terapia hipolipemiante intensiva en el manejo del riesgo cardiovascular ateroesclerótico
}

\author{
Attilio Rigotti $\mathbf{R}^{\mathbf{1}}$, Antonio Arteaga $\mathrm{LI}^{\mathbf{2}}$, Alberto Maiz $\mathbf{G}^{\mathbf{2}}$. \\ Intensive cholesterol lowering \\ therapy to reduce cardiovascular risk
}

Primary and secondary prevention trials have clearly demonstrated that lowering serum cholesterol levels with statins reduces the incidence of cardiovascular events. Recent studies plus post hoc analysis of previous clinical trials show that risk reduction is proportional to the magnitude of LDL cholesterol lowering. Therefore, new recommendations of the National Cholesterol Education Program (USA) have defined a category of patients with very high cardiovascular risk, who should achieve serum LDL cholesterol levels below $70 \mathrm{mg} / \mathrm{dl}$. This proposal will require new and more efficient pharmacologic strategies to attain the increasingly strict therapeutic goals for $\mathrm{LDL}$ cholesterol. This article reviews the clinical studies that support the use of intensive lipid lowering therapy to reduce cardiovascular risk. An effective reduction of serum cholesterol can be obtained using statins in high doses or a combination of hypolipidemic drugs with different mechanisms of action (Rev Méd Chile 2006; 134: 641-8).

(Key w ords: Hydroxymethylglutaryl-CoA reductase in hibitors; Hypercholesterolemia)

Recibido el 17 de agosto, 2005. Aceptado el 20 de octubre, 2005.

Departamentos de ${ }^{1}$ Gastroenterología y ${ }^{2}$ Diabetes, Nutrición y Metabolismo, Facultad de Medicina, Pontificia Universidad Católica de Chile; Santiago, Chile.

L a enfermedad cardiovascular coronaria y cerebrovascular isquémica constituyen la primera causa de mortalidad en Chile ${ }^{1}$. Dentro de las múltiples condiciones predisponentes, la hipercolesterolemia es un factor de riesgo claramente establecido que favorece el desarrollo de ateroesclerosis. De acuerdo con la Encuesta Nacional de Salud 2003, un tercio de la población adulta chilena presenta niveles de colesterol total superiores al rango máximo aceptable, mientras que

Correspondencia a: Dr. Attilio Rigotti. Departamento de Gastroenterología, Facultad de Medicina, Pontificia Universidad Católica. Marcoleta 367, Santiago, Chile. Teléfono: 56-2-6863832. Fax: 56-2-6397780. E mail: arigotti@med.puc.cl
$50 \%$ de la misma, presenta un riesgo alto o muy alto para desarrollar patología cardiovascular ${ }^{2}$, lo que exige la aplicación de medidas terapéuticas efectivas para controlar esta condición proaterogénica. De hecho, múltiples estudios de prevención primaria y secundaria han demostrado, categóricamente, que la terapia hipolipemiante con estatinas en dosis bajas a moderadas disminuye entre 20 y $30 \%$ la incidencia de eventos cardiovasculares a 5 años de seguimiento ${ }^{3}$.

\section{BENEFICIO DE LA TERAPIA HIPOLIPEMIANTE INTENSIVA}

Más recientemente, los estudios Post-Coronary Artery Bypass Graft (Post-CABG) ${ }^{4}$, Atorvastatin 
versus Recascularization Therapy $(A V E R T)^{5}$ y Myocardial Ischemia Reduction with Aggressive Cholesterol Lowering (MIRACL) ${ }^{6}$, han demostrado una menor incidencia de eventos isquémicos en pacientes coronarios que logran alcanzar niveles de colesterol LDL (LDL-C) $\leq 80 \mathrm{mg} / \mathrm{dl}$, comparado con aquéllos que se mantienen con LDL-C entre $120-130 \mathrm{mg} / \mathrm{dl}$. Por ejemplo, el estudio MIRACL 6 estableció que la terapia intensiva con atorvastatina $80 \mathrm{mg} /$ día iniciada precozmente después de un síndrome coronario agudo, redujo en $16 \%$ la aparición de nuevos eventos cardiovasculares a 16 semanas de seguimiento, comparado con un grupo placebo. Este efecto protector cardiovascular se asoció con una reducción del colesterol LDL plasmático desde 124 hasta $72 \mathrm{mg} / \mathrm{dl}$. Este tipo de estudios, asociado al análisis post hoc de protocolos clínicos más antiguos, llevaron a plantear la hipótesis que mientras más se reducen los niveles de colesterol LDL se podría obtener una mayor protección cardiovascular. Aun siendo muy sugerente, este planteamiento fue discutido ${ }^{7} \mathrm{y}$ hasta hace poco tiempo carecía de evidencia derivada de grandes estudios clínicos prospectivos que lo sustentara más sólidamente.

En protocolos clínicos más recientes, esta hipótesis "mientras más se reduce el colesterol LDL es mejor", se ha ido validando hasta establecerse categóricamente. La terapia intensiva con estatinas en dosis altas, que logra niveles de colesterol hasta el rango de 70-80 mg/ dl, tiene un impacto beneficioso adicional con respecto al uso de estatinas en dosis bajas, reduciendo la progresión de las lesiones ateromatosas y mejorando la evolución clínica de la enfermedad isquémica. En primer lugar, el análisis post hoc del estudio Heart Protection Study (HPS), el protocolo clínico con estatinas más grande realizado hasta la fecha, demostró que la terapia con simvastatina mantenía su efecto favorable aun cuando se aplicara en el subgrupo de pacientes con LDL-C basal $<100$ $\mathrm{mg} / \mathrm{dl} 0$ en diabéticos con $\mathrm{LDL}-\mathrm{C}<116 \mathrm{mg} / \mathrm{dl}$, sugiriendo que no existía un límite inferior de niveles de colesterol LDL bajo el cual desaparece el beneficio del uso de estatinas ${ }^{8}$.

Posteriormente, el estudio Reversal of Atherosclerosis with Aggressive Lipid Lowering (REVERSAL) ${ }^{9}$ empleó ultrasonido intracoronario en pacientes con enfermedad coronaria estable para demostrar que el uso de una dosis alta de atorvasta- tina ( $80 \mathrm{mg} /$ día), que alcanzó niveles de colesterol LDL de $79 \mathrm{mg} / \mathrm{dl}$, disminuía significativamente la progresión de las lesiones ateromatosas en un seguimiento hasta 18 meses, en comparación a la terapia con pravastatina (40 mg/día), que sólo redujo el colesterol $\mathrm{LDL}$ a $110 \mathrm{mg} / \mathrm{dl}$. Aunque no fue diseñado para comparar la evolución clínica, este estudio fue el primero en demostrar que una monoterapia intensiva con estatinas era capaz de detener la progresión y, en algunos casos, inducir regresión del daño ateroesclerótico coronario.

El estudio REVERSAL fue corroborado por el estudio Pravastatin or Atorvastatin Evaluation and Infection Therapy-Thrombolysis in Myocardial Infarction 22 (PROVE IT-TIMI 22) ${ }^{10}$, el cual estableció el beneficio de la terapia hipolipemiante agresiva sobre la incidencia de nuevos eventos clínicos, a 2 años plazo, en pacientes con hospitalización reciente por infarto miocárdico o angina inestable. A partir de niveles basales de colesterol LDL de $106 \mathrm{mg} / \mathrm{dl}$, los pacientes del estudio PROVE IT-TIME 22 recibieron atorvastatina $80 \mathrm{mg} /$ día, alcanzando una reducción de 49\% (hasta LDL-C de $62 \mathrm{mg} / \mathrm{dl}$ ), mientras que la terapia con pravastatina $40 \mathrm{mg} /$ día logró una dismimución de $20 \%$ hasta LDL-C de $95 \mathrm{mg} / \mathrm{dl}$. El resultado más importante de este estudio fue una reducción de $16 \%$ de nuevos eventos cardiovasculares mayores, lo cual se manifestó precozmente desde el primer mes de tratamiento. Este estudio sugiere el beneficio de una terapia precoz y agresiva con estatinas en pacientes de alto riesgo con un síndrome coronario agudo. Esta evidencia del beneficio de la terapia intensiva con estatinas en la enfermedad cardiovascular ateroesclerótica, se ha considerado una revolución del paradigma más conservador de la monoterapia con estatinas que se había asumido hasta entonces ${ }^{11}$.

El argumento más reciente que apoya la efectividad de la terapia hipolipemiante intensiva deriva de los resultados del estudio Treating to New Targets $(\mathrm{TNT})^{12}$. Este trabajo demostró que pacientes con enfermedad coronaria estable se beneficiaron adicionalmente (22\% de reducción del riesgo relativo a 6 años de seguimiento), logrando niveles de colesterol LDL de $77 \mathrm{mg} / \mathrm{dl}$ mediante el uso de atorvastatina $80 \mathrm{mg} /$ día, comparados con aquellos que sólo alcanzaron valores promedio de LDL-C de $101 \mathrm{mg} / \mathrm{dl}$, usando una dosis baja de $10 \mathrm{mg} /$ día de la misma estatina. 
NUEVA META TERAPÉUTICA

EN PACIENTES DE ALTO RIESGO CARDIOVASCULAR

Basados en los estudios clínicos prospectivos de intervención disponibles, el National Cholesterol Education Program (NCEP) de Estados Unidos (USA) ha propuesto guías de manejo hipolipemiante cada vez más exigentes para controlar el riesgo cardiovascular, estableciendo que la reducción de los niveles de colesterol LDL debe ser el objetivo terapéutico primario ${ }^{3}$.

Incluso antes de conocerse los resultados del protocolo TNT, la evidencia disponible de algunos de los estudios analizados más arriba (HPS, PROVE IT-TIME 22), junto con los protocolos PROSPER, ALLHAT-LLT y ASCOTT-LLA, llevaron al comité ejecutivo del NCEP a proponer durante el año 2004 una modificación de los objetivos terapéuticos que habían sido publicados previamente ${ }^{13}$. Específicamente, estas nuevas recomendaciones definen una categoría de pacientes de muy alto riesgo cardiovascular, que requieren reducir el colesterol LDL a niveles inferiores de 70 $\mathrm{mg} / \mathrm{dl}$. Esta nueva categoría de riesgo incluye a pacientes con enfermedad coronaria establecida y que presentan: 1) diabetes mellitus, 2) diagnóstico de síndrome metabólico, 3) factores de riesgo mal controlados (especialmente tabaquismo); 0 4) síndrome coronario agudo.

Esta nueva recomendación de terapia hipolipemiante intensiva, junto con el aumento de la frecuencia de los factores de riesgo cardiovascular en la población, generan la necesidad de disponer de estrategias farmacológicas cada vez más efectivas para alcanzar los objetivos terapéuticos de colesterol LDL que se recomiendan en la actualidad. A diferencia de prevención secundaria, es importante dejar en claro que todavía no existen evidencias directas que apoyen la validez del paradigma "mientras más se reduce el colesterol LDL es mejor" en prevención primaria, especialmente en el caso de sujetos con riesgo cardiovascular bajo o moderado. Por otro lado, se debe señalar que la relación entre los niveles de colesterol LDL y el riesgo cardiovascular es semilogarítmica, por lo que una disminución adicional del colesterol LDL desde rangos basales bajos, tiene un menor impacto en la reducción de eventos clínicos ${ }^{13}$, sugiriendo que la relación costo/beneficio del tratamiento pudiera ser mayor en estos pacientes comparados con aquellos sujetos que presentan niveles de colesterol más elevados.

Sin embargo, es importante señalar que no parecen existir efectos adversos derivados de la reducción del LDL a 50-70 mg/dl. De hecho, los recién nacidos presentan niveles de colesterol total de $79 \mathrm{mg} / \mathrm{dl}^{14}$ y de LDL entre $30-70 \mathrm{mg} / \mathrm{dl}^{15}$ en sangre de cordón umbilical. Por otra parte, primates no humanos, así como aborígenes humanos adultos cazadores y recolectores contemporáneos no expuestos al mundo desarrollado, viven normalmente con niveles de colesterol LDL entre 50-75 $\mathrm{mg} / \mathrm{dl}^{15}$, presentando una baja incidencia de enfermedad cardiovascular.

\section{LOGRO DE LOS OBJETIVOS TERAPÉUTICOS} DEL COLESTEROL LDL CON MONOTERAPIA HIPOLPEMIANTE

Las estatinas constituyen actualmente la monoterapia más eficaz para disminuir los niveles de colesterol plasmático ${ }^{3}$. La atorvastatina y la rosuvastatina son los fármacos más potentes disponibles en el mercado, alcanzando reducciones de hasta $60 \%$ en los niveles de colesterol LDL con respecto a los valores pre-tratamiento. Además de la actividad hipocolesterolemiante, las estatinas inducen una amplia gama de efectos pleiotrópicos extralipídicos que contribuirían significativamente a su acción protectora cardiovascular ${ }^{16}$. De hecho, algunos estudios han indicado que la reducción en los niveles de colesterol LDL no explica completamente la mejor evolución de los pacientes que fueron tratados intensamente con estatinas ${ }^{16}$, sugiriendo la contribución de los efectos antiinflamatorios de esta clase de medicamentos.

A pesar de la eficacia y amplia disponibilidad de las estatinas, el logro de las metas de colesterol LDL según la estratificación del riesgo cardiovascular de cada paciente, no ha sido adecuado en la práctica clínica diaria ${ }^{17-21}$. Incluso, el alcance de los objetivos terapéuticos es mucho menor en aquellos pacientes de más alto riesgo cuando estaban vigentes las normas del ATPII ${ }^{17-19}$ y es muy posible que la situación actual sea aún más crítica con las recomendaciones del ATPIII modificado ${ }^{13}$, ya que las metas actuales son aún más exigentes para los pacientes de muy alto riesgo cardiovascular.

A pesar de todas las evidencias disponibles que apoyan su claro beneficio clínico, los médicos aún 
no prescriben adecuadamente la terapia hipolipemiante, conformándose con logros parciales de los objetivos terapéuticos. Esto, posiblemente, se debe al requerimiento de estatinas de alta eficacia en altas dosis, lo que se asocia potencialmente a efectos colaterales hepáticos o musculares que impiden alcanzar todo su potencial terapéutico hipolipemiante y extra-lipídico. Otras razones que pudieran explicar el bajo porcentaje de sujetos que alcanzan los objetivos de colesterol LDL son: el subdiagnóstico de las dislipidemias, la severidad de ellas, la selección inadecuada de fármacos, la mala adherencia al tratamiento y el costo elevado de la terapia.

\section{TERAPIA FARMACOLÓGICA COMBINADA COMO NUEVA ESTRATEGIA HIPOLIPEMIANTE}

La realidad recién mencionada ha llevado al desarrollo del concepto que en muchos pacientes es necesaria una terapia farmacológica hipolipemiante combinada, usando drogas con mecanismos de acción complementarios para el manejo de la hipercolesterolemia ${ }^{22}$.

Combinación de estatinas con ácido nicotínico. El uso del ácido nicotínico asociado a otros fármacos hipolipemiantes (resinas, fibratos y estatinas) ha sido una de las estrategias más exploradas ${ }^{23}$. En este sentido, el HDL Atherosclerosis Treatment Study (HATS $^{24}$ demostró que el tratamiento asociado de niacina con simvastatina redujo los niveles de colesterol LDL desde 132 a $75 \mathrm{mg} / \mathrm{dl}$ y aumentó en $26 \%$ los valores de colesterol HDL, todo lo cual se correlacionó con una mejonía significativa en las lesiones coronarias angiográficas y de los eventos clínicos (reducción dramática de 60-90\%) comparado con el grupo placebo ${ }^{24}$. Así, el primer producto aprobado como terapia combinada en Estados Unidos fue la asociación de niacina de liberación extendida con lovastatina. De hecho, esta terapia combinada (niacina $2.000 \mathrm{mg} /$ lovastatina $40 \mathrm{mg} /$ día) puede reducir los niveles plasmáticos de colesterol LDL hasta en $42 \%$ (similar al efecto de la monoterapia con estatinas en dosis moderada o alta), con un efecto adicional sobre el resto del perfil lipídico, que incluye una importante alza de 32\% en el colesterol HDL y una reducción de 50\% en los triglicéridos, lo que fue superior al uso de estatinas ${ }^{25}$.
A pesar de este favorable efecto normolipemiante, esta terapia combinada tiene como desventaja la presencia de efectos secundarios que pueden limitar la adherencia de algunos pacientes a este plan terapéutico. El ácido nicotínico de liberación extendida presenta una incidencia de bochornos y vasodilatación cutánea generalizada, que va desde 10 a $50 \%$ de los sujetos en los distintos estudios clínicos, lo que puede ser atenuado mediante el uso de ácido acetilsalicílico de liberación rápida en dosis baja, administrada 30 a 60 min antes de ingerir el ácido nicotínico como monoterapia o en combinación con otros fármacos.

Combinación de estatinas con fibratos. Otra altemativa de terapia combinada es la asociación de estatinas con fibratos, especialmente para pacientes de alto riesgo cardiovascular que presentan dislipidemias mixtas no controladas con monoterapia. En general, esta estrategia ha demostrado un efecto hipocolesterolémico similar a la monoterapia con estatinas, pero con un claro efecto sinérgico beneficioso sobre los niveles de colesterol HDL y triglicéridos ${ }^{26}$. Sin embargo, todavía no se han reportado estudios clínicos que evalúen el beneficio sobre las lesiones ateromatosas o sobre la incidencia de eventos clínicos de esta terapia combinada versus la monoterapia con estatinas 0 fibratos. La principal limitación de esta terapia radica en el mayor riesgo de miopatía, el que puede ocurir hasta en $0,1-2,5 \%$ de los pacientes tratados ${ }^{26}$. Dicho riesgo puede reducirse significativamente si se evita el uso de gemfibrozilo en combinación con estatinas y la terapia combinada no se indica en pacientes ancianos, alcohólicos, polimedicados o con patologías asociadas, destacando el daño hepático y la insuficiencia renal ${ }^{27}$.

Combinación de estatinas con ezetimiba. La combinación de estatinas en dosis bajas con ezetimiba es otra interesante alternativa disponible, permitiendo bloquear las dos fuentes básicas de colesterol del organismo: la síntesis endógena y la absorción intestinal de colesterol dietario y biliar. De hecho, la asociación de simvastatina y ezetimiba fue la segunda alternativa de terapia combinada para la hipercolesterolemia aprobada en USA. Esta estrategia permite alcanzar un efecto hipocolesterolémico similar o incluso superior a la monoterapia con estatinas en dosis altas ${ }^{28-33}$. Por ejemplo, la dosis de inicio recomendada de simvastatina $20 \mathrm{mg}$ + ezetimiba 10 
mg/día, redujo los valores de colesterol LDL en 51\%, mientras que la atorvastatina en dosis de inicio (10 $\mathrm{mg} /$ día) sólo los disminuyó en $36 \% 33$. De hecho, para todo el rango de dosis utilizadas, la asociación de simvastatina + ezetimiba fue superior a la monoterapia con atorvastatina, incluyendo el uso de esta última en dosis máxima de $80 \mathrm{mg} /$ día $^{33}$.

Por otro lado, la terapia combinada de estatinas en dosis bajas + ezetimiba, tiene una mayor eficacia para alcanzar los objetivos terapéuticos del colesterol LDL <70 ó $100 \mathrm{mg} / \mathrm{dl}$, cuando se compara con la monoterapia de estatinas de dosis altas ${ }^{33-35}$. Por lo tanto, esta aproximación sería una alternativa terapéutica especialmente relevante para aquellos sujetos de riesgo cardiovascular alto o muy alto, como son los portadores de diabetes mellitus o con patología circulatoria conocida ${ }^{36}$.

Se ha sugerido que las estatinas ejercerían su efecto protector cardiovascular a través de sus efectos pleiotrópicos dosis-dependientes, más allá de su capacidad hipolipemiante. Por lo tanto, se ha planteado que la terapia de inhibición dual con estatinas en dosis bajas y ezetimiba pudiera ejercer un efecto protector cardiovascular menor que el tratamiento intensivo con estatinas en dosis altas $^{37}$. Sin embargo, los estudios que han evaluado los niveles de proteína $C$ reactiva ultrasensible como marcador del efecto extralipídico de los tratamientos hipolipemiantes, han demostrado que la terapia combinada de estatinas en dosis bajas con ezetimiba reducen el estado proinflamatorio en forma similar a las estatinas en dosis altas $^{38,39}$. Estos hallazgos sugerirían que la terapia combinada tendría un efecto beneficioso sobre los eventos clínicos equivalente al tratamiento más agresivo con estatinas. Sin embargo, estudios preliminares del efecto de la terapia combinada versus estatinas en dosis altas sobre la función endotelial, han arrojado resultados contradictorios. La respuesta final a esta pregunta sólo se resolverá cuando finalicen los estudios SEAS (Simvastatin and Ezetimibe in Aortic Stenosis), SHARP (Study of Heart And Renal Protection), ENHANCE (Ezetimibe and Simvastatin in Hypercholesterolemia Enhances Atherosclerosis Regresion) e IMPROVE-IT (Improved Reduction of Outcomes: Vytorin Efficacy International Trial), todo ellos destinados a evaluar el impacto de la terapia combinada de estatinas + ezetimiba sobre la ateroesclerosis subclínica (estudios eco-angio- gráficos de las lesiones ateromatosas) o sobre la incidencia de eventos clínicos a largo plazo.

\section{OTRAS ALTERNATIVAS HIPOLIPEMIANTES EN DESARROLLO}

Actualmente existen en desarrollo una amplia gama de alternativas terapéuticas dirigidas hacia nuevos blancos relacionados con el metabolismo lipídico. Así, se han diseñado inhibidores de la enzima escualeno sintasa ${ }^{40-42}$, la que cataliza el paso metabólico de farnesilpirofosfato a escualeno en la fase terminal de la compleja vía de síntesis de colesterol. Estos compuestos tienen un efecto hipocolesterolémico, incluso superior a las estatinas, cuando se utilizan en roedores o primates no humanos ${ }^{40-42}$, aparentemente sin efectos colaterales mayores.

Por otro lado, la investigación celular y molecular del mecanismo de regulación de la expresión génica del receptor de LDL ha llevado al desarrollo de agonistas farmacológicos de la proteína sensora de los niveles intracelulares de colesterol (SCAP), drogas que reducen en $80 \%$ los niveles de colesterol plasmático cuando se usan en hámsteres hipercolesterolémicos ${ }^{43}$. Adicionalmente, se han descrito nuevos mecanismos posttranscripcionales de regulación del receptor de LDL, los cuales son blancos potenciales para el desarrollo de nuevos fármacos hipolipemiantes $^{44,45}$. La berberina es un compuesto natural que estabiliza los niveles de ARN mensajero del receptor de LDL, aumentando los niveles del producto proteico en las células hepáticas. Este efecto de la berberina se correlaciona con una disminución de $29 \%$ en los niveles de colesterol total en pacientes hipercolesterolémicos ${ }^{44}$.

Además, existen en fase avanzada de desarrollo preclínico y clínico moduladores específicos del metabolismo de HDL (p.e., torcetrapib) ${ }^{46}$. El torcetrapib es inhibidor de la proteína responsable de la transferencia de colesterol éster y triglicéridos (CETP) entre lipoproteínas a nivel intravascular, regulando la remodelación de las HDL y el transporte retrógrado del colesterol ${ }^{46}$. En los primeros estudios humanos se ha demostrado que el torcetrapib aumenta los niveles de colesterol de HDL entre $60-100 \%$ con respecto a los niveles basales, sin apreciarse efectos secundarios significativos $^{47,48}$. Además, la inhibición de CEPT en conejos 
sometidos a una dieta aterogénica, determina un significativo aumento del colesterol de HDL, que se correlacionó con una disminución de las lesiones vasculares ateromatosas ${ }^{49}$. Es posible que el uso clínico rutinario de drogas con efecto predominante sobre el metabolismo de HDL permita incorporar objetivos terapéuticos específicos para el colesterol HDL una vez que se hayan alcanzado las metas de colesterol LDL con el uso de estatinas ${ }^{46}$. Finalmente, también se estudia el posible efecto hipolipemiante de agonistas duales de PPARa y PPARg ${ }^{50}$, ambos receptores nucleares de gran importancia en la regulación del metabolismo lipídico y glucídico.

\section{ADDENDA}

Desde la revisión final de este manuscrito, se han publicado dos nuevos estudios que son consistentes con el beneficio cardiovascular de una terapia hipolipemiante intensiva:

En primer lugar, el estudio IDEAL [JAMA 294: 2437-2445; 2005] demostró que el uso de atorvastatina $80 \mathrm{mg} /$ día versus simvastatina $20-40 \mathrm{mg} /$ día en prevención secundaria, redujo en forma más importante los niveles de colesterol LDL y disminuyó en $11 \%$ la incidencia de cualquier evento coronario mayor, aunque este último resultado alcanzó una significación estadística límite

\section{REFERENCIAS}

1. Causas específicas de defunción en Chile, 2001. Ministerio de Salud, Gobierno de Chile (http:// deis.minsal.cl/ev/mortalidad_general/causas/ as.asp)

2. I Encuesta Nacional de Salud Chile, 2003. Ministerio de Salud, Gobierno de Chile (http:// epi.minsal.cl/epi/html/invest/ENS/ENS.htm)

3. Expert Panel on Detection, Evaluation, and Treatment of High Blood Cholesterol in Adults. Executive Summary of the Third Report of the National Cholesterol Education Program (NCEP) Adult Treatment Panel III (ATPIII). JAMA 2001; 285: 2486-97.

4. Post Coronary Artery Bypass Graft Trial InvestigaTORS. The effect of aggressive lowering of lowdensity lipoprotein cholesterol levels and low-dose anticoagulation on obstructive changes $(p=0,07)$. Sin embargo, este estudio demostró que la mayor reducción del colesterol LDL lograda con el uso de atorvastatina en dosis altas, determinó una menor incidencia de infarto cardíaco no fatal y enfermedad vascular periférica sintomática y menor necesidad de revascularización miocárdica. Cuando se aplicaron los mismos criterios de efectividad que los utilizados en los estudios TNT y PROVE-IT, la terapia hipolipemiante intensiva con atorvastatina del estudio IDEAL redujo en forma similar (13-16\%) y significativa la aparición de nuevos eventos cardiovasculares, comparada con el uso de simvastatina en dosis baja a moderada [JAMA 294: 2492-2494; 2005].

Segundo, y a pesar de algunas limitaciones metodológicas (ausencia de grupo control, exclusión de pacientes con estenosis coronaria avanzada), el estudio ASTEROID [JAMA 295: 1556-1565; 2006] estableció que el tratamiento con la dosis máxima aprobada de rosuvastatina (40 mg/día) permitió alcanzar niveles de colesterol LDL de 61 $\mathrm{mg} / \mathrm{dl}$, lo que se correlacionó con una reducción significativa en el tamaño de la placa ateromatosa. Confirmando lo propuesto por los resultados del protocolo REVERSAL, este estudio constituye la primera evidencia con ultrasonido intracoronario que demuestra una regresión de la ateroesclerosis mediante la aplicación de terapia hipolipemiante intensiva con estatinas.

in saphenous-vein coronary-artery bypass grafts. N Engl J Med 1997; 336: 153-62

5. Pitt B, Waters D, Brown WV, Van Boven AJ, Schwartz L, Title LM et al. Aggressive lipidlowering therapy compared with angioplasty in stable coronary artery disease. Atorvastatin versus Revascularization Treatment Investigators. N Engl J Med 1999; 341: 70-6.

6. Schwartz GG, Olsson AG, EzeKowitz MD, Ganz P, Oliver MF, Waters D et al. Myocardial Ischemia Reduction with Aggressive Cholesterol Lowering (MIRACL) Study Investigators. Effects of atorvastatin on early recurrent ischemic events in acute coronary syndromes: the MIRACL study: a randomized controlled trial. JAMA 2001; 285: 17118.

7. JACOBSON TA. The lower the better» in hypercholesterolemia therapy: a reliable clinical guideline? Ann Intern Med 2000; 133: 549-54. 
8. Heart Protection Study Colnaborative Group. MRC/ BHF Heart Protection Study of cholesterol lowering with simvastatin in 20,536 high-risk individuals: a randomized placebo-controlled trial. Lancet 2002; 360: 7-22.

9. Nissen SE, Tuzcu EM, Schoenhagen P, Brown BG, Ganz P, Vogel RA ET aL. Effect of intensive compared with moderate lipid-lowering therapy on progression of coronary atherosclerosis: a randomized controlled trial. JAMA 2004; 291: 1071-80.

10. Cannon CP, Braunwaid E, McCabe CH, Rader DJ, ROUleau JL, Belder $\mathrm{R}$ et al. Intensive versus moderate lipid lowering with statins after acute coronary syndromes. N Engl J Med 2004; 350: 1495-504.

11. Topol EJ. Intensive statin therapy-a sea change in cardiovascular prevention. N Engl J Med 2004; 350: 1562-4.

12. Larosa JC, Grundy SM, Waters DD, Shear C, Barter P, FRUCHART JC ET AL. Intensive lipid lowering with atorvastatin in patients with stable coronary disease. N Engl J Med 2005; 352: 1425-35.

13. Grundy SM, CleEman JI, Merz CN, Brewer HB JR, Clark LT, HunninghaKe DB et al. Implications of recent clinical trials for the National Cholesterol Education Program Adult Treatment Panel III guidelines. Circulation 2004; 110: 227-39.

14. Arteaga A, Pérez A, Vela P, Pastore H, Torres MC, CAPURRO I ET AL. La colesterolemia en el recién nacido. Rev Méd Chile 1962; 90: 641-5.

15. O'Keefe JH Jr, Cordain L, Harris WH, Moe RM, Vogel R. Optimal low-density lipoprotein is 50 to $70 \mathrm{mg} / \mathrm{dl}$ : lower is better and physiologically normal. J Am Coll Cardiol 2004; 43: 2142-6.

16. Davignon J. Beneficial cardiovascular pleiotropic effects of statins. Circulation 2004; 109: III39III43.

17. Hoerger tJ, Bala MV, Bray JW, Wilcosky TC, LAROSA J. Treatment patterns and distribution of low-density lipoprotein cholesterol levels in treatment-eligible United States adults. Am J Cardiol 1998; 82: 61-5.

18. Pearson ta, Laurora I, Chu H, Kafonek S. The lipid treatment assessment project (L-TAP): A multicenter survey to evaluate the percentages of dyslipidemic patients receiving lipid-lowering therapy and achieving low-density lipoprotein cholesterol goals. Arch Intern Med 2000; 160: 459-67.
19. EUROASPIRE I and II Group; European Action on Secondary Prevention by Intervention to Reduce Events. Clinical reality of coronary prevention guidelines: a comparison of EUROASPIRE I and II in nine countries. Lancet 2001; 357: 995-1001.

20. Ma J, Sehgal NL, Ayanian JZ, Stafford RS. National Trends in Statin Use by Coronary Heart Disease Risk Category. PLoS Med 2005; 2: e123.

21. RoBERTS WC. The underused miracle drugs: the statin drugs are to atherosclerosis what penicillin was to infectious disease. Am J Cardiol 1996; 78: 377-8.

22. BRown AS. Use of combination therapy for dyslipidemia: a lipid clinic approach. Am J Cardiol 2002; 90: 44K-49K.

23. Meyers CD, Kamanna VS, Kashyap ML Niacin therapy in atherosclerosis. Curr Opin Lipidol 2004; 15: 659-65.

24. Brown BG, Zhao XQ, Chatt A, Fisher LD, Cheung MC, Morse JS et aL. Simvastatin and niacin, antioxidant vitamins, or the combination for the prevention of coronary disease. N Engl J Med 2001; 345: 1583-9.

25. Bays HE, Dujovne CA, McGovern ME, White TE, KaSHYAP ML, HuTCHeSON AG et al. Comparison of once-daily, niacin extended-release/lovastatin with standard doses of atorvastatin and simvastatin (the ADvicor Versus Other Cholesterol-Modulating Agents Trial Evaluation [ADVOCATE]). Am J Cardiol 2003; 91: 667-72.

26. Szapary PO, RADER DJ. The triglyceride-high-density lipoprotein axis: an important target of therapy? Am Heart J 2004; 148: 211-21.

27. Pasternak RC, Smith SC Jr, Bairey-Merz CN, Grundy SM, CleEman JI. ACC/AHA/NHLBI Clinical Advisory on the Use and Safety of Statins. Circulation 2002; 106: 1024-8.

28. Gagne C, Gaudet D, Bruckert E. Efficacy and safety of ezetimibe coadministered with atorvastatin or simvastatin in patients with homozygous familial hypercholesterolemia. Circulation 2002; 105: 2469-75.

29. Gagne C, Bays HE, Weiss SR, Mata P, Quinto K, Melino M ET AL. Efficacy and safety of ezetimibe added to ongoing statin therapy for treatment of patients with primary hypercholesterolemia. Am J Cardiol 2002; 90: 1084-91.

30. Davidson MH, McGarRy T, Bettis R, Meiani L, LipKa LJ, LEBEAUT AP ET AL Ezetimibe coadministered with simvastatin in patients with primary hypercholesterolemia. J Am Coll Cardiol 2002; 40: 2125-34. 
31. Kerzner B, Corbe山i J, Sharp S, Lipka LJ, Melani L, LEBEAUT A ET AL. Efficacy and safety of ezetimibe coadministered with lovastatin in primary hypercholesterolemia. Am J Cardiol 2003; 91: 418-24.

32. Davidson MH, Baldantyne CM, Kerzner B, Melani L, SAger PT, LIPKA L ET aL. Efficacy and safety of ezetimibe coadministered with statins: randomized, placebo-controlled, blinded experience in 2382 patients with primary hypercholesterolemia. Int J Clin Pract 2004; 58: 746-55.

33. BaLantyne CM, Abate N, Yuan Z, King TR, PalmisaNo J. Dose-comparison study of the combination of ezetimibe and simvastatin (Vytorin) versus atorvastatin in patients with hypercholesterolemia: the Vytorin Versus Atorvastatin (VYVA) study. Am Heart J 2005; 149: 464-73.

34. Feldman T, Koren M, Insull W Jr, McKenney J, Schrott H, Lewin A et AL. Treatment of high-risk patients with ezetimibe plus simvastatin coadministration versus simvastatin alone to attain National Cholesterol Education Program Adult Treatment Panel III low-density lipoprotein cholesterol goals. Am J Cardiol 2004; 93: 1481-6.

35. Pearson ta, Denke MA, McBride Pe, Battisti WP, Brady WE, Palmisano J. A community-based, randomized trial of ezetimibe added to statin therapy to attain NCEP ATP III goals for LDL cholesterol in hypercholesterolemic patients: the ezetimibe add-on to statin for effectiveness (EASE) trial. Mayo Clin Proc 2005; 80: 587-95.

36. Simons L, Tonkon M, Masana L, Maccubbin D, Shah A, LFE M ET AL. Effects of ezetimibe added to on-going statin therapy on the lipid profile of hypercholesterolemic patients with diabetes mellitus or metabolic syndrome. Curr Med Res Opin 2004; 20: 1437-45.

37. NISSEN SE. High-dose statins in acute comnary syndromes: not just lipid levels. JAMA 2004; 292: 1365-7.

38. Sager PT, Melani L, Lipka L, Strony J, Yang B, SURESH R ET AL. Effect of coadministration of ezetimibe and simvastatin on high-sensitivity Creactive protein. Am J Cardiol 2003; 92: 141418.

39. Sager PT, Capece R, Lipka L, Strony J, Yang B, SURESH R ET AL. Effects of ezetimibe coadministered with simvastatin on C-reactive protein in a large cohort of hypercholesterolemic patients. Atherosclerosis 2005; 179: 361-7.
40. Hiyoshi H, Yanagimachi M, Ito M, Ohtsuka I, YoshidA I, SAEKI T ET AL. Effect of ER-27856, a novel squalene synthase inhibitor, on plasma cholesterol in rhesus monkeys: comparison with 3-hydroxy-3-methylglutaryl-coa reductase inhibitors. J Lipid Res 2000; 41: 1136-44.

41. Amano $Y$, Nishimoto T, Tozawa R, Ishikawa E, Imura Y, SugiYama Y. Lipid-lowering effects of TAK-475, a squalene synthase inhibitor, in animal models of familial hypercholesterolemia. Eur J Pharmacol 2003; 466: 155-61.

42. Nishimoto T, Amano $Y$, Tozawa R, Ishikawa E, Imura Y, YUKIMASA H ET AL. Lipid-lowering properties of TAK-475, a squalene synthase inhibitor, in vivo and in vitro. Br J Pharmacol 2003; 139: 911-8.

43. Grand-Perret T, Bouilot A, Perrot A, Commans S, WALKER M, ISSANDOU M. SCAP ligands are potent new lipid-lowering drugs. Nat Med 2001; 7: 1332-8.

44. Kong W, Wei J, Abidi P, Lin M, Inaba S, Li C et aL. Berberine is a novel cholesterol-lowering drug working through a unique mechanism distinct from statins. Nat Med 2004; 10: 1344-51.

45. MAXwel KN, BResLow JL Proprotein convertase subtilisin kexin 9: the third locus implicated in autosomal dominant hypercholesterolemia. Curr Opin Lipidol 2005; 16: 167-72.

46. Laris MR, Arteaga A, Cuevas A, Rigotti A. El colesterol HDL: ¿Un nuevo objetivo terapéutico en el manejo de las dislipidemias y la ateroesclerosis? Rev Méd Chile 2005, 133: 823-32.

47. Clark RW, Sutfin TA, Ruggeri RB, Wimauer AT, Sugarman ED, Magnus-Ary Itey G et al. Raising high-density lipoprotein in humans through inhibition of cholesteryl ester transfer protein: an initial multidose study of torcetrapib. Arterioscler Thromb Vasc Biol 2004; 24: 490-7.

48. Brousseau ME, Schaefer EJ, Wolfe ML, Bloedon LT, Digenio AG, Ciark RW et al. Effects of an inhibitor of cholesteryl ester transfer protein on HDL cholesterol. N Engl J Med 2004; 350: 1505-15.

49. Morehouse LA, Sugarman ED, Bourassa P-A, Milici AJ. HDL elevation by the CETP-inhibitor torcetrapib prevents aortic atherosclerosis in rabbits. The American Heart Association Scientific Sessions 2004; abstract 1168.

50. Viles-González JF, Choi BG, Fuster V, Badimon JJ. Peroxisome proliferator-activated receptor ligands in atherosclerosis. Expert Opin Investig Drugs 2004; 13: 1393-403. 